\title{
Problème aux dérivées tangentielles discontinues pour une équation elliptique
}

\author{
par W. Pogonzelski $\dagger$ (Warszawa)
}

1. Introduction. Le problème aux limites à dérivée tangentielle, posé pour la première fois par Il. Poincaré [1], consiste en la recherche d'une fonction harmonique ì l'intérieur d'un domaine plan qui vérifie, en tout point de la courbe limitant ce domaine, une relation linéaire entre les valeurs limites: $1^{\circ}$ de la dérivée dans la direction de la normale, $2^{\circ}$ de la dérivée dans la direction de la tangente, $3^{\circ}$ de la fonction elle même.

Le problème de H. Poincaré a fait l'objet des recherches de plusieur's auteurs. Les résultats les plus approfondis ont été obtenus par B. Hvédélidzé [2] et I. Vécoua [3], [4].

L'auteur du présent travail a généralisé le problème de $\mathrm{H}$. Poincaré dans le cas d'une relation non linéaire entre les valeurs limites des dérivées au bord du domaine donné (voir [5]). Ensuite, dans les travaux [6] et [7], l'auteur a étudić le problème non linéaire aux limites aux dérivées tangentielles pour les équations elliptique et parabolique d'une forme générale dans l'espace à $n$ dimensions. Ces problèmes ont été résolus par la métlıode topologique de Schauder. Dans la publication [8] ce problème est résolu par la méthode des approximations successives.

Dans le présent travail l'auteur étudiera le problème aux dérivées tangentielles discontinues pour une équation elliptique dans le plan, i. coefficients hölderiens.

2. Énoncé du problème. Soit dans le plan une ligne fermée de Jordan $L_{0}$ limitant le domaine borné $\Omega$. Soient à l'intérieur du domaine $\Omega$ des lignes fermées $L_{1}, L_{2}, \ldots, L_{q}$, disjointes, limitant avec la ligne $L_{0}$ un domaine $S_{0}$. L'ensemble des lignes $L_{1}, \ldots, L_{q}$ peut être vide, alors on a $S_{0}=\Omega$. Soient en outre à l'intérieur du domaine $S_{0}$ les ares furmés et disjoints $l_{1}, l_{2}, \ldots, l_{q^{\prime}}$. On admet que les lignes $L_{0}, \ldots, L_{q}$ et les ares $l_{1}, \ldots, l_{q^{\prime}}$ ont des tangentes continues en tout point. Soit ensuite un ensemble fini de points $C_{1}, C_{2}, \ldots, C_{n}$, composé de toutes les extrémités des $\operatorname{arcs} l_{1}, \ldots, l_{Q^{\prime}}$ et en outre, peut être, de points choisis arbitrairement 
sur ces arcs mêmes et sur les lignes fermées $L_{0}, \ldots, L_{q}$ (pas nécessairement sur chacune). Ces points peuvent être arbitrairement ordonnés. On admet que la ligne $L_{0}$ a une direction positive dans le plan orienté et que les lignes fermées intérieures $L_{1}, \ldots, L_{q}$ ont les directions négatives. Les directions des ares non fermés $l_{1}, \ldots, l_{q^{\prime}}$ sont choisies arbitrairement. Désignons par $L$ l'ensemble de points

$$
L=\sum_{\nu=0}^{q} L_{\nu}+\sum_{\nu=1}^{q^{\prime}} l_{\nu} .
$$

Soit une équation elliptique dans le plan, de la forme

$$
\begin{aligned}
\Psi(u) & =\sum_{i, j=1}^{2} a_{i j}(X) \frac{\partial^{2} u}{\partial x_{i} \partial x_{j}}+\sum_{j=1}^{2} b_{j}(X) \frac{\partial u}{\partial x_{j}}+c(X) u \\
& =F\left(X, u, \frac{\partial u}{\partial x_{1}}, \frac{\partial u}{\partial x_{2}}\right)
\end{aligned}
$$

Nous admettons les hypothèses suivantes.

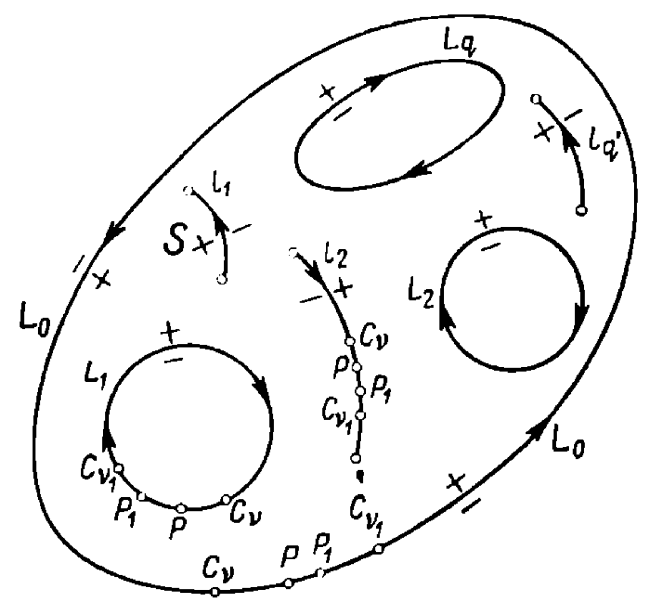

Fig. 1

I. Les coefficients $a_{i j}(X), b_{j}(X), c(X)$ sont définis en tout point $X\left(x_{1}, x_{2}\right)$ du domaine fermé $\Omega+L_{0}$ et vérifient les conditions de Hölder

$$
\begin{aligned}
\left|a_{i j}(X)-a_{i j}\left(X^{\prime}\right)\right| & \leqslant \text { const }\left|X X^{\prime}\right|^{h}, \\
\left|b_{j}(X)-b_{j}\left(X^{\prime}\right)\right| & \leqslant \text { const }\left|X X^{\prime}\right|^{h}, \\
\left|c(X)-c\left(X^{\prime}\right)\right| & \leqslant \text { const }\left|X X^{\prime}\right|^{h},
\end{aligned}
$$

où $\left|X X^{\prime}\right|$ désigne la distance euclidienne des deux points $X, X^{\prime}$ du domaine $\Omega, h$ est une constante positive non supérieure à l'unité. 
II. La forme quadratique

$$
\sum_{i, j=1}^{2} a_{i j}(X) Z_{i} Z_{j}
$$

est définie - positive dans le domaine $\Omega+L_{0}$.

III. La fonction réelle $F\left(X, u_{0}, u_{1}, u_{2}\right)$ est définie dans la région ouverte:

$$
X \in S, \quad-\infty<u_{v}<+\infty \quad(v=0,1,2)
$$

où $S$ désigne l'ensemble de tous les points du domaine $S_{0}$ non situés sur les $\operatorname{arcs} l_{v}$.

La fonction $F$ vérifie l'inégalité suivante:

$$
\left|F\left(X, u_{0}, u_{1}, u_{2}\right) i<-\frac{M_{F}}{\left|X P_{X}\right|^{p}}+M_{F}^{\prime} \sum_{\nu=0}^{2}\right| u_{v} \mid
$$

où $P_{\boldsymbol{X}}$ désigne un point de l'ensemble $L$ le plus rapproché du point intérieur $X, M_{F}$ et $M_{F}^{\prime}$ sont des constantes positives, la constante non négative $p$ est inférieure à l'unité $(0 \leqslant p<1)$. En outre la fonction $F$ vérifie une condition de Hölder-Lipschitz

$$
\begin{aligned}
\left|F\left(X, u_{0}, u_{1}, u_{2}\right)-F\left(X^{\prime}, u_{0}^{\prime}, u_{1}^{\prime}, u_{2}^{\prime}\right)\right| \leqslant C\left(S^{*}\right) \mid & \left.X X^{\prime}\right|^{h_{F}}+ \\
& +k_{F} \sum_{\nu=0}^{2}\left|u_{v}-u_{\nu}^{\prime}\right|^{h_{F}^{\prime}}
\end{aligned}
$$

en tout domaine fermé $S^{*}$ situé à l'intérieur de l'ensemble $S$. Le coefficient positif $C\left(S^{*}\right)$ dépend du domaine $S^{*}$ et peut être non borné; le coefficient positif $k_{F}$ ne dépend pas du domaine $S^{*}$; les constantes positives $h_{F}$ et $h_{F}^{\prime}$ ne sont pas supérieures à l'unité.

$\Gamma$. Nous supposons que l'angle $\Delta\left(P, P_{1}\right)$ entre les tangentes en deux points arbitraires $P, P_{1}$ de chaque arc $l_{v}$ ou ligne $I_{v}$ vérifie l'inégalité

$$
\Delta\left(P, P_{1}\right) \leqslant \text { const }\left|P P_{1}\right|^{h} L,
$$

où la constante positive $h_{L}$ n'est pas supérieure à l'unité.

V. La fonction réelle donnée $g(P)$, définie en tout point $P$ sur $L$, différent des points de discontinuité $C_{v}$, vérifie les inégalités

$$
|g(P)|<M_{g}, \quad\left|g(P)-g\left(P_{1}\right)\right|<\frac{k_{g}\left|P P_{1}\right|^{\mu}}{\left[\left|P C_{g}\right|\left|P_{1} C_{v_{1}}\right|\right]^{\mu}},
$$

où l'on admet que les points $P, P_{1}$ sont situés à l'intérieur d'un même $\operatorname{arc}{\widehat{C} C_{v}}_{n}$ joignant deux points de discontinuité voisins sur $L$ et que 
$P_{1} \in \overparen{P C}_{\nu_{1}}, M_{g}$ et $k_{0}$ sont des constantes positives; la constante $\mu$ vérifie la condition $0<\mu<1$. Conformément à la définition donnée par l'auteur dans le travail [9], la fonction $g(P)$ appartient à la classe $\mathfrak{S}_{0}^{\prime \prime}$.

VI. La fonction réelle donnée $G(P, u, v)$, définie dans la région

$$
P \in L-\sum C_{v}, \quad-\infty<u<+\infty, \quad \cdots-\infty<v<+\infty,
$$

vérifie les inégalités

$$
\begin{aligned}
& |G(P, u, v)| \leqslant M_{G}(|u|+|v|)+\frac{M I_{G}^{\prime}}{\prod_{v=1}^{n}\left|P C_{v}\right|^{\alpha}} ; \\
& \left|G(P, u, v)-G\left(P^{\prime}, u^{\prime}, v^{\prime}\right)\right| \leqslant M_{G}\left[\left|u-u^{\prime}\right|^{h_{\alpha}}+\left|v-v^{\prime}\right|\right]+\frac{k_{\alpha}\left|P P^{\prime}\right|^{\mu}}{\left[\left|P C_{v}\right|\left|P^{\prime} C_{\nu_{1}}\right|\right]^{a+\mu}} \\
& \left(P, P^{\prime} \in{\overparen{C_{v} C_{v_{1}}}}, P^{\prime} \in \overparen{P C}_{v_{1}}\right)
\end{aligned}
$$

où $M_{G}, M I_{G}^{\prime}, k_{G}, h_{G}$ sont des constantes positives $\left(h_{G} \leqslant 1\right)$. Par conséquent, la fonction $G(P, u, v)$ appartient à la classe $\mathfrak{S}_{a}^{u}$ relativement à la variable $P$; on admet que

$$
0 \leqslant \alpha<1, \quad 0<\mu<1, \quad a+\mu<1 .
$$

Le problème aux limites que nous nous proposons d'étudier consiste en la recherche d'une fonction $u(X)$ qui vérifie l'équation (1) en tout point intérieur $X$ de l'ensemble $S$ et dont les dérivées ont des valeurs limites vérifiant l'égalité

$$
\frac{d u}{d T_{P}}+g(P) u(P)=G\left[P, u(P), u_{s}(P)\right]
$$

en tout point $P \in L-\sum_{\nu=1}^{n} C_{v} ; d u / d T_{P}$ désigne la valeur limite, au point $P$, de la dérivée transversale

$$
\frac{d u}{d T_{P}}=\lim _{X \rightarrow P} \sum_{i, j=1}^{n} a_{i j}(X) \cos \left(N_{P}, x_{j}\right) \frac{\partial u}{\partial x_{j}} \quad\left(P \neq C_{v}\right)
$$

où la direction positive de la normale $N_{P}$ à tout arc $l_{\nu}$ est dans la même relation avec sa direction que les normales aux lignes frontières $L_{0}, L_{1}, \ldots, L_{n}$ intérieures au domaine $S_{0}$, avec les directions de ces lignes. Ensuite $u_{s}(P)$ désigne la valeur limite de la dérivée dans la direction positive de la tangente au point $P$ :

$$
u_{s}(P)=\lim _{X \rightarrow P} \frac{d u(X)}{d s_{P}} \quad\left(P \neq C_{v}\right)
$$


La limite (11) concerne les points $X$ situés du côté positif de tout arc $l_{a}$ et la limite (12) se rapporte à ceux qui sont situés des deux côtés de tout are (excepté $L_{a}$ ). On demande que les limites (11) et (12) ne dépendent pas de la manière dont le point $X$ tend vers le point $P \neq C_{\nu}$. Outre la condition (10), nous demandons que les dérivées de la fonction cherchée $u(X)$ vérifient une inégalité de la forme

$$
\left|\frac{\partial u(X)}{\partial x_{j}}\right|<\frac{\text { const }}{\prod_{\nu=1}^{p}\left|X C_{\nu}\right|^{\theta}}
$$

où la constante positive $\theta$ est inférieure à l'unité. Nous admettons encore l'hypothèse suivante.

VII. Ise problène aux limites homogène:

$$
\frac{d v}{d T_{P}}+g(P) v=0, \quad P \in L-\sum_{v=1}^{n} C_{v}
$$

pour l'équation homogène $\Psi(v)=0$, en conservant la condition (13), n'a qu'une solution nulle $v=0$.

3. Les formules et les théorèmes fondamentaux. Conformément aux recherches connues de Levi, continuées par Gevrey [10] et Giraud [11] (voir aussi le travail [12]), la solution fondamentale de l'équation (1) est donnée par la formule

$$
\Gamma(X, Y)=w^{\bar{Y}}(X, Y)+\iint_{\Omega^{\prime}} w^{Z}(X, Z) \Phi(Z, Y) d Z
$$

(en négligeant les termes réguliers) pour tout couple $X, Y$ de points différents d'un domaine mesurable $\Omega^{\prime} \supset \Omega$. La quasi-solution est donnée par la formule

$$
w^{Z}(X, Y)=\log \left[\sum_{i, j=1}^{2} a^{i j}(Z)\left(x_{i}-\xi_{i}\right)\left(x_{j}-\xi_{j}\right)\right]
$$

où $a^{i j}(I)$ désignent les éléments de la matrice inverse de la matrice $\left\{a_{i j}(Z)\right\}, x_{i}$ et $\xi_{i}$ sont les coordonnées des points $X$ et $Y$. La fonction $\Phi$ dans la formule (14) est la solution d'une équation de Fredholm. Dans notre travail nous nous appuierons sur les propriétés suivantes de quelques intégrales de l'équation elliptique.

THÉoR亡̀me 1. Si la densité $\varphi(P)$ de simple couche, définie pour $P \in L-\sum C_{v}$, appartient d̀ la classe $\mathfrak{H}_{a}^{\mu}$, c'est-à-dire si elle vérifie les inégalités

$$
|\varphi(P)|<\frac{M_{\omega}}{\prod_{\nu=1}^{n}\left|P C_{\nu}\right|^{a}}, \quad\left|\varphi(P)-\varphi\left(P_{1}\right)\right| \leqslant \frac{k_{\varphi}\left|P P_{1}\right|^{\mu}}{\left[\left|P C_{\nu}\right|\left|P_{1} C_{\nu_{1}}\right|\right]^{\alpha+\mu}}
$$


en vertu de notre travail [9] $(0 \leqslant \alpha<1, \alpha+\mu<1,0<\mu<1)$, $P$ et $P_{1}$ étant situés à l'intérieur d'un même arc ${\widehat{C_{v} C_{p_{1}}}}_{\text {et }} P_{1} \in \widehat{P C}_{v_{1}}$, alors le potentiel de simple couche, étalée sur les lignes $L$ est défini par la formule

$$
U(X)=\int_{L} \Gamma(X, Q) \varphi(Q) d Q
$$

(dQ désigne l'élément de longueur au point Q), a les proprietés suivantes:

1) La fonction (17) possède des dérivées premières et secondes continues vérifiant l'équation

$$
\begin{gathered}
\Psi[U(X)]=0 \\
\text { en tout point intérieur } X \text { de l'ensemble } S=S_{0}-\sum_{p=1}^{q^{\prime}} l_{v}
\end{gathered}
$$

2) La fonction (17) a une limite déterminée

$$
U(P)=\lim _{X \rightarrow P} U(X)=\int_{L} \Gamma(P, Q) \varphi(Q) d Q
$$

en tout point $P$ sur $L$.

3) Il existe une valeur limite de la dérivée transversale du potentiel (17), relative au côté positif de l'arc, exprimée par la formule

$$
\frac{d U(P)}{d T_{P}}=\frac{\pi}{\sqrt{\operatorname{det}\left|a^{i j}(P)\right|}} \varphi(P)+\int_{L} \frac{d \Gamma(P, Q)}{d T_{P}} \varphi(Q) d Q
$$

en tout point $P \in L$ différent des points $C_{1}, \ldots, C_{n}$; la fonction sous le signe intégral admet la limitation

$$
\left|\frac{d \Gamma(P, Q)}{d T_{P}}\right|<\frac{\text { const }}{|P Q|^{1-h_{1}}}, \quad \text { où } h_{1}=\min \left(h, h_{L}\right) .
$$

4) $\Pi$ existe une valeur limite bilatérale de la dérivée tangentielle du potentiel (17), déterminée par une intégrale singulière au sens de Cauchy

$$
U_{s}(P)=\lim _{X \rightarrow P} U_{s_{P}}(X)=\int_{L} \Gamma_{s}(P, Q) \varphi(Q) d Q
$$

en tout point $P$ sur $L$, différent des points $C_{\nabla} ;$ la fonction sous le signe intégral admet une singularité forte, notamment elle est comparable à la fonction $(s-\sigma)^{-1}$, où $s$ et $\sigma$ désignent les abscisses curvilignes des points $P$ et $Q$.

Les propriétés 1 et 2 sont évidentes, puisqu'elles n'exigent que l'intégrabilité de la fonction $\varphi$. La propriété 3 est vraie sous l'hypothèse suffisante que la fonction $\varphi(Q)$ soit intégrable et continue au point $\boldsymbol{P}$.

La propriété 4 est vérifiée, puisque la fonction $\varphi(Q)$ est intégrable et safisfait à une simple condition de Hölder dans un voisinage suffisamment petit du point $P$ ne contenant pas les points de discontinuité $C_{v}$. Observons encore que la limite (20) ne dépend pas de la manière dont le point intérieur $X$ tend vers le point $P \neq C_{v}$ (voir le travail [13] de l'auteur). 
THÉORÈme 2. Si la fonction $\varphi(Q)$, définie et intégrable sur $L-\sum C_{\eta}$, vérifie une inégalité

$$
|\varphi(Q)|<\frac{M_{\varphi}}{\prod_{n=1}^{n}\left|Q C_{\nu}\right|^{a}} \quad(0 \leqslant \alpha<1)
$$

où $M_{\varphi}$ est une constante positive, alors les fonctions définies par les intégrales

$$
\begin{aligned}
& J_{1}(P)=\int_{L} \Gamma(P, Q) \varphi(Q) d Q \\
& J_{2}(P)=\int_{L} \frac{d \Gamma(P, Q)}{d T_{P}} \varphi(Q) d Q
\end{aligned}
$$

vérifient les inégalités suivantes

$$
\begin{gathered}
\left|J_{1}(P)\right|<\text { const } M_{\varphi}, \\
\left|J_{1}(P)-J_{1}\left(P_{1}\right)\right| \leqslant \frac{\text { const }}{\left[\left|P C_{v}\right| \mid P_{1} C_{v_{1}}\right]^{\theta}}, \\
\left|J_{2}(P)\right|<\frac{\text { const } M_{\varphi}}{\prod_{v=1}^{n}\left|P C_{v}\right|^{a}} \\
\left|J_{2}(P)-J_{2}\left(P_{1}\right)\right| \leqslant \frac{\text { const } M_{\varphi}\left|P P_{1}\right|^{\theta h_{1}}}{\left[\left|P C_{v}\right|\left|P_{1} C_{v_{1}}\right|\right]^{a+\theta h_{1}}}
\end{gathered}
$$

à l'intérieur de tout arc $\widehat{C_{\nu} C_{v_{1}}}$, ò̀ $\theta$ est une constante positive, arbitrairement inférieure à l'unité, $h_{1}=\min \left(h, h_{L}\right)$, les coefficients positifs const. aux numérateurs ne dépendant pas de la fonction $\varphi$.

Par conséquent, d'après le travail [9], les fonctions (22') et (22') appartiennent aux classes

$$
J_{1}(P) \in \mathfrak{S}_{0}^{\theta}, \quad J_{2}(P) \in \mathfrak{S}_{\alpha}^{\theta h_{1}}
$$

relativement aux points de discontinuité $C_{v}$. Le théorème cité sera important pour notre étude, mais, sa démonstration étant analogue à celles de la théorie classique du potentiel, nous ne la reproduirons pas.

Théonème 3. Si la densité $\varphi(Q)$ appartient à la classe $\mathfrak{S}_{a}^{\mu}(a>0)$, o'est-à-dire si elle vérifie les inégalités (16), alors les dérivées du potentiel (17) de simple couche vérifient des inégalités de la forme

$$
\left|D_{x j}(X)\right|<\frac{c M_{\varphi}+c^{\prime} k_{\varphi}}{\prod_{v=1}^{n}\left|X C_{v}\right|^{a}} \quad(j=1,2)
$$

en tout point $X$ a l'intérieur de l'ensemble $S ; c$ et $c^{\prime}$ sont des constantes positives, indépendantes de la fonction $\varphi$. 
Démonstration. Il suffit d'étadier l'intégrale de la dérivée de la quasi-solution étendue à l'arc $\widehat{C}_{v_{v_{1}}}$

$$
J(X)=\int_{C_{v} C_{n}} w_{x_{j}}(X, Q) \varphi(Q) d Q
$$

au voisinage du point de discontinuité $C_{v}$, puisque la partie restante concerne la fonction $\bar{w}_{x}$ à singularité faible. Soit $C_{v} T_{0}$ la demi-tangente issue du point $C_{v}$, sur laquelle sont situées les projections orthogonales des points de l'arc $\widehat{C}_{\nu} C_{v_{1}}$ au voisinage du point $C_{\nu}$. Soient ensuite deux demi-droites $C_{\nu} T_{1}$ et $C_{v} T_{2}$ situées des deux côtés de la demi-tangente $C_{v} T_{0}$ et faisant avec elle des angles aigus égaux. Considérons un arc $\overparen{C, W}$, partie de l'arc ${\overparen{C_{p}}}_{v_{1}}$, situé à l'intérieur de l'angle $T_{1} C_{v} T_{2}$, et soit un triangle $\Delta$ limité par les demi-droites $C_{v} T_{1}, C_{7} T_{2}$ et une perpendiculaire à la demi-tangente $C, T_{0}$, passant par le point $W$ - extrémité de l'arc $\widehat{C_{v} W}$. Nous admettons que l'arc $\overparen{C, W}$ est suffisamment petit pour que: 1) la tangente en tout point $P$ de l'arc $\widehat{C_{v} W}$ (extrémités comprises) fasse un angle aigu avec la tangente $C_{v} T_{0}$ plus petit que l'angle entre $C_{\nu} T_{0}$ et $C_{v} T_{1}$; 2) la perpendiculaire à la demi-droite $C, T_{0}$, passant par un point arbitraire $X \mathrm{du}$ triangle $\Delta$, coupe l'arc $\widehat{C_{v} W}$ en un seul point $P$. Considérons encore le triangle $\Delta^{\prime}$ limité par les demi-droites $C_{\nu} T_{1}, C_{v} T_{2}$ et une perpendiculaire à $C_{v} T_{0}$, passant par un point $W^{\prime}$ de l'arc $\widehat{C_{v} W}$ qui le divise en deux parties $\widehat{C, W^{\prime}}$ et $\widehat{W^{\prime} W}$ de longueurs égales.

Pour limiter l'intégrale (26), supposons que $X$ soit on point arbitraire du triangle $\Delta^{\prime}$, non situé sur l'arc $\widehat{C_{\nu} W}$, et décomposons l'intégrale (26) en une somme d'intégrales

$$
J(X)=J^{\left(C_{P_{0}}\right)}(X)+J^{\left(P_{0} W\right)}(X)+J^{\left(W C_{v_{1}}\right)}(X)
$$

étendues aux arcs $\widetilde{C, P}_{0}, \widehat{P_{0} W}, \widehat{W C}_{v_{1}}$, où $P_{0}$ est un point de l'arc $\widehat{C_{v} W}$ tel que les arcs $\overparen{C_{v} P}$ et $\widehat{P P}_{0}$ aient la même longueur, $P$ désigne le point d'intersection de l'arc $\widehat{C_{v} W^{\prime}}$ avec la perpendiculaire à la demi-tangente $C_{v} T_{0}$ passant par le point $X \in \Delta^{\prime}$.

Écrivons maintenant la première des intégrales (32) sous la forme suivante:

$$
J^{\left(C_{v} P_{0}\right)}(X)=\int_{C_{v} P_{0}} w_{x_{j}}^{Q}(X, Q)[\dot{\varphi}(Q)-\varphi(P)] d Q+\varphi(P) \int_{C_{v} P_{0}} w_{x_{j}}^{Q}(X, Q) d Q
$$

La première de ces intégrales, d'après la formule (15) et l'inégalité (24), admet la limitation suivante: 


$$
\begin{aligned}
\mid \int_{C_{\nu} P_{\mathbf{0}}} w_{x,}^{Q}(X, Q)[\varphi(Q) & -\varphi(P)] d Q \mid \\
& <\int_{C_{v} P} \frac{\operatorname{const} k_{\varphi} d Q}{\left|Q C_{v}\right|^{a+\mu}|P Q|^{1-\mu}}+\int_{P P_{0}}^{\cdot} \frac{\text { const } k_{\varphi} d Q}{\left|P C_{v}\right|^{a+\mu}|P Q|^{1-\mu}},
\end{aligned}
$$

puisque le rapport des distances $|P Q| /|X Q|$ admet une borne supérieure pour $X \in \Delta^{\prime}$. Il en résulte

$$
\left|\int_{C_{\nu} P_{0}} w_{x_{j}}^{Q}(X, Q)[\varphi(Q)-\varphi(P)] d P\right|<\frac{\text { const } k_{\varphi}}{\left|P C_{\nu}\right|^{a}}<\frac{\text { const } k_{\varphi}}{\left|X C_{v}\right|^{\alpha}}
$$

en remarquant que le rapport $\left|X C_{v}\right| /\left|P C_{\nu}\right|$ admet une borne supérieure pour $X \in \Delta^{\prime}$.

D'après notre travail [1.3], la seconde des intégrales (28) est bornée si $X \rightarrow P$; par conséquent, d'après l'inégalité (21), nous aurons

$$
\left|\varphi(P) \underset{C_{\nu} P_{0}}{\int_{P_{0}}} w_{x j}^{Q}(X, Q) d Q\right|<\frac{\operatorname{const} M_{\varphi}}{\left|X C_{\nu}\right|^{a}}
$$

En rapprochant les résultats (30) et (31), nous obtenons la limitation suivante

$$
\left|J^{\left(C_{v} P_{0}\right)}(X)\right|<\frac{\widetilde{c} M_{\varphi}+\widetilde{c}^{\prime} k_{\varphi}}{\left|X C_{v}\right|^{\alpha}}
$$

pour $X \in \Delta^{\prime}$. Pour étudier la seconde des intégrales (27), désignons par $s$ et $\sigma$ les longueurs des arcs $\overparen{C_{v} P}$ et $\overparen{C_{v} Q}$ et appliquons l'inégalité

$$
\frac{\sigma-s}{\sigma} \geqslant \frac{1}{2}, \quad \text { si } \quad \sigma \geqslant 2 s
$$

nous aurons alors

$$
\begin{aligned}
\left|J^{\left(P_{0} W\right)}(X)\right| & <\text { const } M_{\varphi} \int_{P_{0} W} \frac{d Q}{|P Q||Q C|^{a}} \\
& <\operatorname{const} M_{\varphi} \int_{2 s}^{\left|C_{\nu} W\right|} \frac{d \sigma}{\sigma^{a+1}}<\frac{\text { const } M_{\varphi}}{\mid X} \frac{\left.C_{v}\right|^{a}}{}
\end{aligned}
$$

La troisième des intégrales (27) est évidemment bornée et admet la limitation

$$
\left|J^{\left(W C_{v_{1}}\right)}(X)\right|<\text { const } M_{\varphi}
$$


si $X \in \Delta^{\prime}$. En réunissant les limitations (32), (33), (34), nous aurons

$$
|J(X)|<\frac{\widetilde{c}_{1} M_{\varphi}+\widetilde{c}_{1}^{\prime} k_{\varphi}}{\left|X C_{v}\right|^{a}}
$$

si $X \in \Delta^{\prime}$.

Il reste à étudier le cas où le point $X$ est situé dans le domaine $D$ qui est la partie du voisinage du point $C_{v}$, extérieure à l'angle convexe entre les demi-droites $C_{v} T_{1}$ et $C_{v} T_{2}$. On peut montrer, par une analyse élémentaire, dans le cas où le point $Q$ est situé sur l'are $\widehat{C_{v} W}$ et le point $X$ dans le domaine $D$, que les trois distances $\left|X C_{\bullet}\right|,|X Q|,\left|Q C_{v}\right|$ satisfont à l'inégalité

$$
\frac{|X Q|}{\left|\bar{X} C_{v}\right|+\left|Q C_{v}\right|} \geqslant \sin \frac{\vartheta}{2}
$$

où $\vartheta$ désigne une borne inférieure positive de la mesure de l'angle aigu compris entre le vecteur $\overrightarrow{C_{v} Q}$ et les demi-droites $C_{v} T_{1}$ et $C_{v} T_{2}$. Il en résulte pour la partie de l'intégrale (26) étendue à l'arc $\widehat{C_{v} W}$, si $X \in D$, la limitation

$$
\begin{aligned}
\left|\int_{C, W} w_{x,}^{Q}(X, Q) \varphi(Q) d Q\right| & \\
& <\left(\sin \frac{\vartheta}{2}\right)^{-1} \int_{C_{\nu} W}^{\cdot} \frac{\text { const } M_{\varphi} d Q}{\left|C_{\nu} Q\right|^{a}\left[\left|X C_{\nu}\right|+\left|Q C_{\nu}\right|\right]}<\frac{\text { const } M_{\varphi}}{\left|X C_{\nu}\right|^{a}} .
\end{aligned}
$$

En rapprochant les limitations (35) et (37), nous arrivons à la conclusion (25) du théorème 3 .

THÉORÈmE 4. Si la densité $\varphi(Q)$ appartient à la classe $\mathfrak{S}_{a}^{\mu}(0<a<1$, $0<\mu<h, a+\mu<1)$ c'est-à-dire si elle vérifie les inégalités (16), alors la fonction limite (20) de la dérivée tangentielle du potentiel (17) de simple couche vérifie les inégalités

$$
\begin{gathered}
\left|U_{s}(P)\right|<\frac{c_{1} M_{\varphi}+c_{2} k_{\varphi}}{\prod_{v=1}^{n}\left|P C_{v}\right|^{n}} \\
\left|U_{s}(P)-U_{s}\left(P_{1}\right)\right|<\frac{\left(c_{1}^{\prime} M_{\varphi}+c_{2}^{\prime} k_{\varphi}\right)\left|P P_{1}\right|^{\mu}}{\left[\left|P C_{\nu}\right| \mid P_{1} C_{v_{1}}\right]^{a+\mu}}
\end{gathered}
$$

(ò̀ $c_{1}, c_{2}, c_{1}^{\prime}, c_{2}^{\prime}$ sont des constantes positives, indépendantes de la fonction $\varphi$ ) $\grave{a}$ l'intérieur de tout are $\widehat{C}_{\nabla_{v_{1}}}$, cette fonction appartient donc aussi $\dot{a}$ la classe $\mathfrak{S}_{a}^{\mu}$, de mêmes paramètres a et $\mu$ que la classe de la densité $\varphi$.

Pour démontrer le théorème, il suffit d'étudier l'intégrale

$$
F(P)=\int_{\sigma_{v} C_{v_{1}}} \Gamma_{s}(P, Q) \varphi(Q) d Q
$$


où $P$ est situé à l'intérieur ou à l'extérieur de l'arc ${\widehat{C_{v} C_{\nu_{1}}}}_{\text {. En désignant }}$ par $s$ et $\sigma$ les abscisses curvilignes des points $P$ et $Q$ sur $\widehat{C}_{v} C_{v_{1}}$, nous pouvons écrire la dérivée tangentielle $\Gamma_{s}$ sous la forme

$$
\Gamma_{s}(P, Q)=\frac{\Phi(s, \sigma)}{s-\sigma}
$$

done

$$
F(P)=F(s)=\int_{C_{v} C_{p_{1}}} \frac{\Phi(s, \sigma)}{s-\sigma} \varphi(\sigma) d \sigma
$$

On montre sans peine, en s'appuyant sur les propriétés (2) et (6), que la fonction $\Phi(s, \sigma)$ satisfait à une condition de Hölder de la forme

$$
\begin{array}{r}
\left|\Phi(s, \sigma)-\Phi\left(s_{1}, \sigma_{1}\right)\right|<\mathrm{const}\left[\left|s-s_{1}\right|^{\theta h_{1}}+\left|\sigma-\sigma_{1}\right|^{\theta h_{1}}\right] \\
\left(\text { où } h_{1}=\min \left(h, h_{L}\right), 0<\theta<1\right)
\end{array}
$$

sur tout arc faisant partie de l'ensemble $L$. Par conséquent, on peut étudier l'intégrale (41), dans les cas où le point $P$ est situé soit à l'extérieur soit à l'intérieur de l'arc $\widehat{C, C}_{v_{1}}$, tout à fait comme dans nos travaux [14], [15], [16] consacrés aux propriétés des fonctions de classe $\mathfrak{S}_{a}^{h}$ définies sur un système arbitraire d'arcs dans le plan de la variable complexe. On arrive ainsi aux conclusions (38). Observons encore que la limitation de la forme

$$
|F(P)|<\frac{\operatorname{const} M_{\varphi}}{\left|P C_{\nabla}\right|^{\alpha}\left|P C_{v_{1}}\right|^{\alpha}}
$$

dans le cas où le point $P$ est situé à l'extérieur de l'arc $\widehat{C}_{\nabla} C_{v_{1}}$, résulte d'ailleurs de nos considérations à la fin de la démonstration du théorème précédent.

THÉORÈmE 5. Si la fonction $\varrho(\bar{Y})$, définie et intégrable dans l'ensemble $\mathbf{S}$, vérifie l'inégalité

$$
|\varrho(Y)|<\frac{M_{\varrho}}{\prod_{p=1}^{n}\left|Y C_{v}\right|^{a}}+\frac{M_{e}^{\prime}}{\left|\bar{Y} P_{Y}\right|^{p}},
$$

où $p$ et a sont des constantes non négatives, inférieures à l'unité, $M_{Q}$ et $M_{Q}^{\prime}$ des constantes positives, $P_{F}$ désigne le point de l'ensemble $L$ le plus rapproché du point intérieur $Y \in S$, alors le potentiel de charge plane

$$
\nabla(X)=\iint_{S} \Gamma(X, Y) \varrho(Y) d Y
$$


(dY désigne l'élément d'aire au point $Y$ ) possède les propriétés suivantes:

1) Le potentiel (44) et ses dérivées tendent vers des limites déterminées

$$
\begin{aligned}
\lim _{X \rightarrow P} V(X) & =\iint_{S} \Gamma(P, Y) \varrho(Y) d Y=V(P), \\
\lim _{X \rightarrow P} \nabla_{x_{j}}(X) & =\iint_{S} \Gamma_{x_{j}}(P, Y) \varrho(Y) d Y=V_{x_{j}}(P),
\end{aligned}
$$

si le point $X$ tend d'une façon arbitraire vers un point $P$ sur $L$, différent des points de discontinuité $C_{\text {. }}$.

2) Le potentiel (44) et ses dérivées admettent les limitations

$$
|\nabla(X)|<c_{0} M_{Q}+c_{0}^{\prime} M_{e}^{\prime}, \quad\left|V_{x_{j}}(X)\right|<c_{1} M_{e}+c_{1}^{\prime} M_{e}^{\prime}
$$

dans l'ensemble $S+L$, où les constantes positives $c_{0}, c_{0}^{\prime}, c_{1}, c_{1}^{\prime}$ ne dépendent pas de la fonction $\varrho(\bar{Y})$.

3) Le potentiel (44) vérifie dans l'ensemble $S+L$ une condition de Lipschitz

$$
\left.\left|V(X)-V\left(X_{1}\right)\right| \leqslant 2\left(c_{1} M_{e}+c_{1}^{\prime} M_{e}^{\prime}\right) ! X X_{1}\right\} .
$$

4) La fonction limite de la dérivée du potentiel (44) vérifie à l'intérieur de tout arc $C_{v_{1}} C_{v_{1}}$ une condition de Hölder

$$
\left|V_{x_{j}}(P)-V_{x_{j}}\left(P_{1}\right)\right| \leqslant \frac{\widetilde{c}_{0} M_{Q}+\widetilde{c}_{0}^{\prime} M_{o}^{\prime}}{\prod_{\nu=1}^{n}\left|P C_{p}\right|^{a}\left|P_{1} C_{p_{1}}\right|^{a}}\left|P P_{1}\right|^{0}
$$

où $\theta=1-p$, si $0<p<1$, et $\theta$ est une constante positive arbitraire, inférieure à l'unité, si $p=0$.

5) Les dérivées du potentiel (44) vérifient une condition de Hölder

$$
\left|V_{x_{j}}(X)-\nabla_{x_{j}}\left(X_{1}\right)\right|<C\left(\mathcal{S}^{*}\right)\left|X X_{1}\right|^{\theta}
$$

dans tout domaine fermé $S^{*}$ situé dans l'ensemble $S, C\left(S^{*}\right)$ est un coefficient positif, dépendant du domaine $S^{*}$ et $d u$ choix de la constante positive $\theta<1$.

Les propriétés citées résultent de nos considérations dans le travail [15].

THÉoRÈmE 6. Si la fonction $\varrho(Y)$, définie et intégrable dans l'ensemble $S$, vérifie l'inégalité

$$
|\varrho(Y)|<\frac{M_{\varrho}}{\prod_{\nu=1}^{n}\left|Y C_{\nu}\right|^{a}}+\frac{M_{e}^{\prime}}{\left|Y P_{P}\right|^{p}} \quad(0 \leqslant a<1 ; 0 \leqslant p<1)
$$

et une condition de Hölder

$$
\left|\varrho(Y)-\varrho\left(Y_{1}\right)\right|<k\left(S^{*}\right)\left|Y Y_{1}\right|^{h_{\varrho}} \quad\left(0<h_{\varrho} \leqslant 1\right)
$$


dans un domaine fermé $S^{*} C S$, alors le potentiel de charge plane (44) vérifip l'équation de Poisson

$$
\Psi[V(X)]=\frac{2 \pi}{\sqrt{\operatorname{det}} \overline{\left|a^{i j}(\bar{X})\right|}} \varrho(X)
$$

en tout point intérieur $X$ du domaine $S^{*}$.

La démonstration de ce théorème résulte des considérations présentées dans les travaux connus de Gevrey et Giraud (voir aussi le travail [12]).

4. Résolution du problème. Nous allons chercher la solution du problème aux limites discontinues proposé sous forme de la somme

$$
u(X)=\iint_{S} \Gamma(X, Y) F_{1}\left[Y, u(Y), \frac{\partial u}{\partial \xi_{1}}, \frac{\partial u}{\partial \xi_{2}}\right] d Y+\int_{L} \Gamma(X, Q) \varphi(Q) d Q
$$

d'un potentiel de charge plane et d'un potentiel de simple couche de densité inconnue $\varphi(Q)$ sur $L$. On a posé

$$
F_{1}\left(Y, u, u_{1}, u_{2}\right)=(2 \pi)^{-1} \sqrt{\operatorname{det}\left|a^{i j}(\bar{Y})\right|} \boldsymbol{F}\left(Y, u, u_{1}, u_{2}\right) .
$$

En demandant que la fonction (53) vérifie la condition limite (10), on arrive, en vertu des théorèmes 1 et 5 , à l'équation

$$
\begin{gathered}
\text { (55) } \frac{\pi}{\sqrt{\operatorname{det}\left|a^{i j}(P)\right|}} \varphi(P)+\int_{\Sigma}\left[\frac{d \Gamma(P, Q)}{d T_{P}}+g(P) \Gamma(P, Q)\right] \varphi(Q) d Q+ \\
+\iint_{S}\left[\frac{d \Gamma(P, Y)}{d T_{P}}+g(P) \Gamma(P, Y)\right] F_{1}\left[Y, u(Y), \frac{\partial u}{\partial \xi_{1}}, \frac{\partial u}{\partial \xi_{2}}\right] d Y \\
=G\left\{P, u(P), \iint_{S} \Gamma_{s}(P, Y) F_{1}\left[Y, u(Y), \frac{\partial u}{\partial \xi_{1}}, \frac{\partial u}{\partial \xi_{2}}\right] d Y+\int_{L} \Gamma_{s}(P, Q) \varphi(Q) d Q\right\}
\end{gathered}
$$

sous l'hypothèse que les conditions des théorèmes 1 et 5 soient satisfaites. L'équation (55) doit être vérifiée en tout point $P$ sur $L$, différents des points $C_{v}$.

Notre problème est donc ramené à l'étude du système de deux équations intégro-différentielles (53), (55) à deux fonctions inconnues: $u(X)$ dans l'ensemble $S$ et $\varphi(Q)$ dans l'ensemble $L-\sum C_{\nu}$.

Le système (53), (55) étant irrésoluble par les méthodes de l'Analyse classique, nous l'étudierons par la méthode topologique basée sur le théorème suivant de J. Schauder [17]:

,Toute transformation continue d'un ensemble convexe, borné, fermé et contenu dans un espace de Banach, en un sous-ensemble compact a au moins un point invariant". 
Cette étude exigera des considérations plus délicates, à cause de la singularité forte d'une intégrale dans l'équation (55) et du fait que la fonction $\varphi(P)$ et les dérivées de la fonction $u(X)$ ne sont pas bornées dans les voisinages des points $C_{v}$.

Considérons done le système des quatre équations intégrales singulières suivantes

$$
\begin{array}{r}
u_{\boldsymbol{r}}(X)=\iint_{S} \Gamma_{\boldsymbol{v}}(X, Y) F_{1}\left[Y, u_{0}(Y), u_{1}(Y), u_{2}(Y)\right] d Y+ \\
\quad+\int_{L} \Gamma_{v}(X, Q) \varphi(Q) d Q \quad(v=0,1,2), \\
\frac{\pi}{\sqrt{\operatorname{det}\left|a^{i j}(P)\right|}} \varphi(P)+\int_{L}\left[\frac{d \Gamma(P, Q)}{d T_{P}}+g(P) \Gamma(P, Q)\right] \varphi(Q) d Q+ \\
+\int_{S} \int\left[\frac{d \Gamma(P, Y)}{d T_{P}}+g(P) \Gamma(P, Y)\right] F_{1}\left[Y, u_{0}(Y), u_{1}(Y), u_{2}(Y)\right] d Y \\
=G\left[P, u_{0}(P), \bar{u}_{s}(P)\right]
\end{array}
$$

à quatre fonctions inconnues $u_{0}(X), u_{1}(X), u_{2}(X), \varphi(P)$, où l'on a désigné

$$
\bar{u}_{8}(P)=\iint_{S} \Gamma_{s}(P, Y) F_{1}\left[Y, u_{0}(Y), u_{1}(Y), u_{2}(Y)\right] d Y+\int_{L} \Gamma_{8}(P, Q) \varphi(Q) d Q,
$$

$$
\Gamma_{0}=\Gamma, \quad \Gamma_{,}=\Gamma_{x_{0}} \quad(v=1,2) .
$$

Soit maintenant un espace fonctionnel $\Lambda$ composé de tous les systèmes

$$
\left[u_{0}(X), u_{1}(X), u_{2}(X), \varphi(P)\right]
$$

de fonctions continues réelles, définies soit dans l'ensemble ouvert $X \in S$, soit dans l'ensemble $P \in L-\Sigma C$, et vérifiant les inégalités

$$
\begin{gathered}
\left|X P_{X}\right|^{\nu} \prod_{j=1}^{n}\left|X C_{j}\right|^{\beta+h_{\varphi}}\left|u_{\nu}(X)\right|<\infty \quad(\nu=0,1,2), \\
\prod_{j=1}^{n}\left|P C_{j}\right|^{\beta+h_{\varphi}}|\varphi(P)|<\infty
\end{gathered}
$$

où $\beta$ est une constante égale à l'exposant donné $a$ dans la propriété (9) si $\alpha>0$, et une constante positive arbitraire, inférieure à l'unité, si $\alpha=0$, $h_{\varphi}$ est une constante positive, fixées arbitrairement de façon qu'on ait

$$
\beta+h_{\varphi}<1, \quad 0<h_{\varphi}<\min \left(\mu, h, h_{L}, h_{G}, 1-p\right),
$$

la constante positive $\mu$ figurant dans les propriétés (7) et (9); $\gamma$ est une constante positive, inférieure à l'unité, arbitrairement fixée. On définit, 
comme d'habitude, la somme de deux points de l'espace $A$ et le produit d'un point par un nombre réel, par les formules

$$
\begin{aligned}
{\left[u_{0}, u_{1}, u_{2}, \varphi\right]+\left[u_{0}^{\prime}, u_{1}^{\prime}, u_{2}^{\prime}, \varphi^{\prime}\right] } & =\left[u_{0}+u_{0}^{\prime}, u_{1}+u_{1}^{\prime}, u_{2}+u_{2}^{\prime}, \varphi+\varphi^{\prime}\right] \\
\lambda\left[u_{0}, u_{1}, u_{2}, \varphi\right] & =\left[\lambda u_{0}, \lambda u_{1}, \lambda u_{2}, \lambda \varphi\right] .
\end{aligned}
$$

Ensuite on définit la norme d'un point $U=\left[u_{0}, u_{1}, u_{2}, \varphi\right]$ par la formule

$$
\begin{aligned}
\|U\|=\max _{0 \leqslant \nu \leqslant 2} \sup _{X \in S}\left[\left|X P_{X}\right|^{\gamma} \prod_{j=1}^{n}\left|X C_{j}\right|^{\beta+h}\left|u_{\nu}(X)\right|\right]+ & \\
& +\sup _{P \in L}\left[\prod_{j=1}^{n}\left|P C_{j}\right|^{\beta+h_{\varphi}}|\varphi(P)|\right]
\end{aligned}
$$

et la distance des deux points $U, \nabla$ par la formule

$$
\delta(U, \nabla)=\|U-\nabla\| \text {. }
$$

On peut montrer sans peine que l'espace ainsi défini est complet, il est donc un espace de Banach.

Considérons maintenant dans l'espace $\Lambda$ l'ensemble $E$ de tous les points $U=\left[u_{0}(X), u_{1}(X), u_{2}(X), \varphi(P)\right]$ vérifiant les inégalités

$$
\begin{gathered}
\prod_{j=1}^{n}\left|X C_{j}\right|^{\beta}\left|u_{p}(X)\right| \leqslant \varrho_{1} \quad(v=0,1,2), \\
\prod_{j=1}^{n}\left|P C_{j}\right|^{\beta}|\varphi(P)| \leqslant \varrho_{9}, \\
{\left[\left|P C_{j}\right|\left|P_{1} C_{j_{1}}\right|\right]^{\beta+h_{\varphi}}\left|\varphi(P)-\varphi\left(P_{1}\right)\right| \leqslant x_{\varphi}\left|P P_{1}\right|^{h_{\varphi}},}
\end{gathered}
$$

où $\varrho_{1}, \varrho_{2}, \varkappa_{\varphi}$ sont des constantes positives, arbitrairement fixées, on a ensuite $P, P_{1} \in{\widehat{C_{j} C_{j_{1}}}}_{1}, \quad P_{1} \in \widehat{P C}_{j_{1}}$. Les fonctions $\varphi(P)$ appartiennent donc à la classe $\mathfrak{S}_{a}^{h}$.

L'ensemble $E$ est évidemment fermé et convexe. Tenant compte de la forme des équations intégrales (56), transformons l'ensemble $E$ par les relations suivantes

$$
\begin{aligned}
& v_{v}(X)=\iint_{S} \Gamma_{v}(X, Y) F_{1}\left[Y, u_{0}(Y), u_{1}(Y), u_{2}(Y)\right] d Y+ \\
& +\int_{L} \Gamma_{\nu}(X, Q) \varphi(Q) d Q \quad(v=0,1,2), \\
& \frac{\pi}{\sqrt{\operatorname{det}\left|a^{i j}(P)\right|}} \psi(P)+\int_{L}\left[\frac{d \Gamma(P, Q)}{d T_{P}}+g(P) \Gamma(P, Q)\right] \psi(Q) d Q \\
& =-\iint_{S}\left[\frac{d \Gamma(P, Y)}{d T_{P}}+g(P) \Gamma(P, Y)\right] F_{1}\left[Y, u_{0}(Y), u_{1}(Y), u_{2}(Y)\right] d Y+ \\
& +G\left[P, v_{0}(P), \bar{u}_{s}(P)\right] \text {. }
\end{aligned}
$$


Cherchons les conditions pour que ces relations fassent correspondre à tout point $U=\left[u_{0}, u_{1}, u_{2}, \varphi\right]$ de l'ensemble $E$ un point déterminé $\nabla=\left[v_{0}, v_{1}, v_{2}, \psi\right]$ du même ensemble. Remarquons pour cela, en vertu de la conclusion (30) du théorème 4 et des inégalités (62), qu'on a la limitation

$$
\left|\int_{L} \Gamma_{v}(X, Q) \varphi(Q) d Q\right|<\frac{c \varrho_{2}+c^{\prime} \varkappa_{\varphi}}{\prod_{j=1}^{n}\left|X C_{j}\right|^{\beta}}
$$

où les constantes positives $c$ et $c^{\prime}$ ne dépendent pas de la fonction $\varphi$. Ensuite, d'après les inégalités (4) et (62), nous aurons la limitation

$$
\left|F_{1}\left[Y, u_{0}(Y), u_{1}(Y), u_{2}(Y)\right]\right|<\frac{3 c_{a} M_{F}^{\prime} \varrho_{1}}{\prod_{j=1}^{n}\left|Y C_{j}\right|^{\beta}}+\frac{c_{a} M_{F}}{\left|Y P_{Y}\right|^{p}}
$$

où $c_{a}=(2 \pi)^{-1} \sup \sqrt{\operatorname{det}\left|a^{i i}(X)\right|}$. Nous en concluons, en vertu du théorème 5 , que les fonctions $v_{,}(X)$, déterminées par la première des formules (63), sont définies et continues dans l'ensemble ouvert $S$ et vérifient l'inégalité suivante

$$
\left|v_{v}(X)\right|<\frac{a_{1} M_{F}+a_{2} M_{F}^{\prime} \varrho_{1}+c \varrho_{2}+c^{\prime} \varkappa_{\varphi}}{\prod_{j=1}^{n}\left|X C_{j}\right|^{\beta}} \quad(v=0,1,2)
$$

où les nouvelles constantes positives $a_{1}, a_{2}$ ne dépendent pas des fonctions $F, u_{\nu}, \varphi$. Pour étudier la fonction $\psi(P)$, remarquons qu'elle est une solution de l'équation de Fredholm de la forme

$$
\frac{\pi}{\sqrt{\operatorname{det}\left|a^{i j}(\bar{X})\right|}} \psi(P)+\int_{L}\left[\frac{d \Gamma(P, Q)}{d T_{P}}+g(P) \Gamma(P, Q)\right] \psi(Q)=f(P)
$$

où $f(P)$ est une fonction déterminée par le second membre de l'équation (63). Le noyau de l'équation (67) a une faible singularité précisée par l'inégalité $\left(19^{\prime}\right)$. Cherchons d'abord une limitation pour la fonction $f(P)$. Observons que, d'après les théorèmes 3 et 5 , la fonction $\left(56^{\prime}\right)$ vérifie l'inégalité

$$
\left|\bar{u}_{s}(P)\right|<\frac{a_{1}^{\prime} M_{F}+a_{2}^{\prime} M_{F}^{\prime} \varrho_{1}+\bar{c} \varrho_{2}+\bar{c}^{\prime} \varkappa_{\varphi}}{\prod_{j=1}^{n}\left|P C_{j}\right|^{a}}
$$

analogue à l'inégalité (66). Il en résulte, en vertu de l'hỵpothèse (9), l'inégalité

$$
\begin{aligned}
& \left|G\left[P, u_{0}(P), \bar{u}_{s}(P)\right]\right| \\
& <\frac{M_{G}\left[\left(a_{1}+a_{1}^{\prime}\right) M_{F}+\left(a_{2}+a_{2}^{\prime}\right) M_{F}^{\prime} \varrho_{1}+(c+\bar{c}) \varrho_{2}+\left(c^{\prime}+\bar{c}^{\prime}\right) \varkappa_{\psi}\right]+M_{G}^{\prime}}{\prod_{j=1}^{n}\left|P C_{j}\right|^{\beta}}
\end{aligned}
$$


et ensuite, en tenant compte de l'inégalité (65) et du théorème 5 , nous aurons la limitation

$$
|f(P)|<\frac{a_{3}\left(M_{F}+M_{F}^{\prime} \varrho_{1}\right)\left(M_{\theta}+a_{3}^{\prime}\right)+a_{4} M_{g}\left(M_{F}+M_{F}^{\prime} \varrho_{1}+\varrho_{2}+x_{\varphi}\right)+M_{G}^{\prime}}{\prod_{j=1}^{n}\left|P C_{j}\right|^{\beta}}
$$

où $a_{3}, a_{9}^{\prime}, a_{4}$ sont des constantes positives, indépendantes des fonctions $F, G, g, u_{v}, \varphi$. En vertu de l'hypothèse VII à la page 37 concernant le problème homogène, l'équation (67) a donc une solution unique, donnée par le premier théorème de Fredholm,

$$
\psi(P)=\pi^{-1} \sqrt{\operatorname{det} \mid a^{i j}(P)}\left|f(P)+\pi^{-1} \int_{L} \mathfrak{N}(P, Q) \sqrt{\operatorname{det} \mid a^{i j}(Q)}\right| f(Q) d Q
$$

continue en tout point $P \neq C_{j}$ où $\mathfrak{R}(P, Q)$ est la somme de plusieurs noyaux itérés et du noyau résolvant. Nous en concluons que la fonction (71) vérifie une inégalité

$$
|\psi(P)|<\frac{b\left(M_{F}+M_{F}^{\prime} \varrho_{1}\right)\left(M_{g}+b^{\prime}\right)+b^{\prime \prime} M_{G}\left(M_{F}+M_{F}^{\prime} \varrho_{1}+\varrho_{2}+x_{\varphi}\right)+M_{G}^{\prime}}{\prod_{j=1}^{n}\left|P C_{j}\right|^{\beta}}
$$

de la même forme que (70); $b, b^{\prime}, b^{\prime \prime}$ sont des constantes positives. Cherchons maintenant l'inégalité de Hölder pour la fonction $\psi(P)$. Dans ce but, en s'appuyant sur les théorèmes 3,5 et sur les inégalités (62), (65), nous aurons pour la fonction $\left(56^{\prime}\right)$ une inégalité de Hölder

$$
\left|\bar{u}_{\theta}(P)-\bar{u}_{s}\left(P_{1}\right)\right|<\frac{\left[c_{1}^{\prime \prime}\left(M_{F}+M_{F}^{\prime} \varrho_{1}\right)+c_{1}^{\prime} \varrho_{2}+c_{2}^{\prime} \varkappa_{\varphi}\right]\left|P P_{1}\right|^{h_{\varphi}}}{\left[\left|P C_{v}\right| \mid P_{1} C_{v_{1}}\right]^{\beta+h_{\varphi}}}
$$

où $P, P_{1} \in \overparen{C, C}_{n_{1}}, \quad P_{1} \in \overparen{P C}_{n_{1}}$. En s'appuyant maintenant sur la seconde des équations (63), sur les inégalités (7), (9), (65), (72), (73) et sur les théorèmes 2, 5, nous arriverons à l'inégalité demandée de la forme

$$
\left|\psi(P)-\psi\left(P_{1}\right)\right|<\left\{b _ { 1 } ( M _ { g } + k _ { \sigma } + b _ { 1 } ^ { \prime } ) \left[\left(M_{F}^{\prime} \varrho_{1}+M_{F}\right)\left(M_{G}+M_{g}+k_{\sigma}+b_{1}^{\prime \prime}\right)+\right.\right.
$$

$$
\left.\left.+M_{G}\left(\varrho+\varkappa_{\varphi}\right)+M_{G}^{\prime}\right]+b_{2} M_{G}\left(M_{F}+M_{F}^{\prime} \varrho_{1}+\varrho_{2}+\varkappa_{\Phi}\right)+k_{G}\right\} \frac{\left|P P_{1}\right|^{h_{\Phi}}}{\left[\left|P C_{v}\right|\left|P_{1} C_{v_{1}}\right|\right]^{\beta+h_{\varphi}}}
$$

où $b_{1}, b_{1}^{\prime}, b_{1}^{\prime \prime}, b_{2}$ sont des constantes positives, indépendantes des fonctions $\mathrm{du}$ problème. Les fonctions transformées $\psi(\boldsymbol{P})$ appartiennent donc à la classe $\mathfrak{S}_{\beta}^{h_{\varphi}}$, de même que les fonctions $\varphi(P)$. En comparant les inégalités obtenues (66), (72), (74) aux inégalités (62), nous pouvons dire que l'ensemble $E^{\prime}$, transformé de l'ensemble $E$ par les relations (63), fera partie 
de cet ensemble si les constantes du problème $M_{F}, M_{F}^{\prime}, M_{G}, M_{G}^{\prime}, k_{G}, M_{g}, k_{\sigma}$ sont suffisamment petites pour que les inégalités suivantes soient vérifiées:

$$
\begin{array}{r}
a_{1} M_{F}+a_{2} M_{F}^{\prime} \varrho_{1}+c \varrho_{2}+c^{\prime} \varkappa_{\varphi} \leqslant \varrho_{1}, \\
b\left(M_{F}+M_{F}^{\prime} \varrho_{1}\right)\left(M_{g}+b^{\prime}\right)+b^{\prime \prime} M_{G}\left(M_{F}+M_{F}^{\prime} \varrho_{1}+\varrho_{2}+\varkappa_{\varphi}\right)+M_{G}^{\prime} \leqslant \varrho_{2}, \\
b_{1}\left(M_{g}+k_{g}+b_{1}^{\prime}\right)\left[\left(M_{F}^{\prime} \varrho_{1}+M_{F}\right)\left(M_{G}+M_{g}+k_{\theta}+b_{1}^{\prime \prime}\right)+\right. \\
\left.+M_{G}\left(\varrho_{2}+x_{\varphi}\right)+M_{G}^{\prime}\right]+b_{2} M_{G}\left(M_{F}+M_{F}^{\prime} \varrho_{1}+\varrho_{2}+x_{\varphi}\right)+k_{G} \leqslant \varkappa_{\varphi} .
\end{array}
$$

Les conditions (75) étant vérifiées, nous démontrerons encore les deux lemmes suivants.

Lemane 1. La transformation de l'ensemble $E$ par les relations (63) est continue dans l'espace $\Lambda$.

Démonstration. Soit une suite arbitraire $\left\{U^{(m)}\right\}$ de points $U^{(m)}=\left[u_{0}^{(m)}, u_{1}^{(m)}, u_{2}^{(m)}, \varphi^{(m)}\right]$ de l'ensemble $E$ convergente vers un point $U=\left[u_{0}, u_{1}, u_{2}, \varphi\right]$ du même ensemble au sens de la norme (60). Nous arons done

$$
\begin{aligned}
\left\|U^{(m)}-U\right\|=\max _{\nu} \sup _{X \in S}\left[\left|X P_{X}\right|^{\gamma} \prod_{j=1}^{n}\left|X C_{j}\right|^{\beta+h_{\Phi}}\left|u_{\nabla}^{(m)}(X)-u(X)\right|\right]+ \\
+\sup _{P \in L}\left[\prod_{j=1}^{n}\left|P C_{j}\right|^{\beta+h_{\varphi}}\left|\varphi^{(m)}(P)-\varphi(P)\right|\right] \rightarrow 0
\end{aligned}
$$

si $m \rightarrow \infty$. Conformément à la définition de la continuité d'une transformation, il est nécéssaire et suffisant de démontrer que la suite de points $\nabla^{(m)}=\left\lfloor v_{0}^{(m)}, v_{1}^{(m)}, v_{2}^{(m)}, \psi^{(m)}\right\rfloor$ transformés par les relations (63), tend vers le point $\nabla=\left[v_{0}, v_{1}, v_{2}, \psi\right]$ de l'ensemble $E^{\prime}$, correspondant au point limite $U=\left[u_{0}, u_{1}, u_{2}, \varphi\right]$ par les mêmes relations (63), c'est-à-dire que

$$
\lim _{m \rightarrow \infty}\left\|\nabla^{(m)}-\nabla\right\|=0 \text {. }
$$

Considérons pour cela la différence

$$
\begin{aligned}
& v_{v}^{(m)}(X)-v_{v}(X) \\
& \quad=\iint_{S} \Gamma_{v}(X, Y)\left\{F_{1}\left[Y, u_{0}^{(m)}(Y), u_{1}^{(m)}(Y), u_{2}^{(m)}(Y)\right]-\right. \\
& \left.-F_{1}\left[Y, u_{0}(Y), u_{1}(Y), u_{2}(Y)\right]\right\} d Y+\int_{L} \Gamma_{v}(X, Q)\left[\varphi^{(m)}(Q)-\varphi(Q)\right] d Q .
\end{aligned}
$$

La première intégrale $\Delta_{1}$ de cette somme vérifie, d'après la limitation

$$
\left|\Gamma_{v}(X, Y)\right|<\frac{\text { const }}{|X Y|}
$$


et l'hypothèse (5), l'inégalité suivante:

$$
\begin{aligned}
& \left|\Delta_{1}(X)\right|<\mathrm{const} \iint_{S} \frac{k_{F} \sum_{,=0}^{2}\left|u_{\nu}^{(m)}(\bar{Y})-u_{\nabla}(\bar{Y})\right|^{h_{F}^{\prime}}}{|\overline{X Y}|} d Y
\end{aligned}
$$

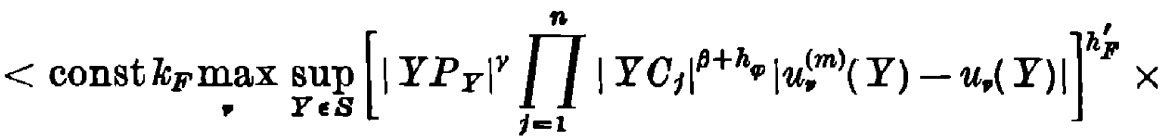

$$
\begin{aligned}
& \times \iint_{S} \frac{d Y}{|X Y|\left|Y P_{Y}\right|^{\nu} \prod_{j=1}^{n}\left|Y C_{j}\right|^{h_{j}^{\prime}\left(\beta+h_{\varphi}\right)}} .
\end{aligned}
$$

Il en résulte, d'après l'hypothèse (76),

$$
\lim _{m \rightarrow \infty} \sup _{X \in S}\left[\left|X P_{X}\right|^{\gamma}\left|\Delta_{1}(X)\right|\right]=0 \text {. }
$$

Pour étudier la seconde intégrale $\Delta_{2}$ de la somme (77), remarquons que, d'après les inégalités (62) et le théorème 4, cette intégrale vérifie l'inégalité

$$
\left|\Delta_{2}(X)\right|<\frac{c_{1} \varrho_{2}+c_{2} \varkappa_{\varphi}}{\prod_{j=1}^{n}\left|X C_{j}\right|^{\beta}}
$$

où les constantes positives $c_{1}, c_{2}$ ne dépendent pas des fonctions $\varphi^{(m)}, \varphi$.

Considérons maintenant un ensemble de cercles disjoints $K_{j}$ centrés aux points de discontinuité $C_{j}$. D'après l'inégalité (80), à tout nombre positif $\varepsilon$ on peut faire correspondre un rayon $r(\varepsilon)$ de ces cercles suffisamment petit pour que l'inégalité

$$
\left|X P_{X}\right|^{\nu} \prod_{j=1}^{n}\left|X C_{j}\right|^{\beta+h_{\varphi}}\left|\Delta_{2}(X)\right|<\varepsilon
$$

soit vraie en tout point $X$ de l'ensemble $S$ situé dans les cercles $K_{j}(\varepsilon)$.

Ajoutons que le rayon $r(\varepsilon)$ est suffisamment petit pour que toute circonférence du cercle $K_{j}$ coupe l'arc correspondant ${\overparen{C_{j} C_{f_{1}}}}_{\text {en }}$ un seul point. Les cercles $K_{j}(\varepsilon)$ étant fixés, étudions l'intégrale $\Delta_{2}(X)$ lorsque le point $X$ de l'ensemble $S$ est extérieur aux cercles $K_{j}$ :

$$
X \in \omega(\varepsilon)=S-\left(\sum_{j=1}^{n} K_{1}\right) \times S .
$$

Dans ce but écrivons

$$
\begin{aligned}
\Delta_{2}(X)= & {\left[\varphi^{(m)}\left(P_{X}^{\prime}\right)-\varphi\left(P_{X}^{\prime}\right)\right] \int_{L} \Gamma_{\nu}(X, Q) d Q+} \\
& +\int_{L} \Gamma_{\nu}(X, Q)\left\{\left[\varphi^{(m)}(Q)-\varphi^{(m)}\left(P_{X}^{\prime}\right)\right]-\left[\varphi(Q)-\varphi\left(P_{X}^{\prime}\right)\right]\right\} d Q
\end{aligned}
$$


$P_{x}^{\prime}$ étant le point de l'ensemble $L$, non intérieur aux cercles $K_{f}$, qui est le plus rapproché du point $X \in \omega(\varepsilon)$. La première intégrale dans la somme (83) étant bornée, nous en concluons, en vertu de l'hypothèse (76), qu'an nombre arbitraire $\varepsilon$ on peut faire correspondre un indice $N(\varepsilon)$ tel qu'on ait

$$
\left|X P_{X}\right|^{\nu} \prod_{j=1}^{n}\left|X C_{j}\right|^{\beta+h_{\varphi}}\left|\varphi^{(m)}\left(P_{X}^{\prime}\right)-\varphi\left(P_{X}^{\prime}\right)\right|\left|\int_{L} \Gamma_{v}(X, Q) d Q\right|<\frac{\varepsilon}{2}
$$

si $m>N(\varepsilon), X \in \omega(\varepsilon)$. Pour étudier le second terme $\bar{\Delta}_{2}(X)$ de la somme (83), considérons un arc $\widehat{l}^{\prime} l^{\prime \prime}$ sur ${\widehat{C_{j} C_{j_{1}}}}_{1}$, contenant le point $P_{X}^{\prime}$, tel que les longueurs des arcs ${\widehat{l^{\prime} P_{X}^{\prime}}}^{\prime},{\widetilde{P_{X}^{\prime}}}^{\prime \prime}$ soient égales, si les points $l^{\prime}$ et $l^{\prime \prime}$ sont extérieurs aux cercles $K_{j}$; dans le cas où une extrémité $l^{\prime}$ est située sur la circonférence du cercle $K_{i}$, on admet que la longueur de l'arc $\widehat{l}^{\prime} P_{X}^{\prime}$ est

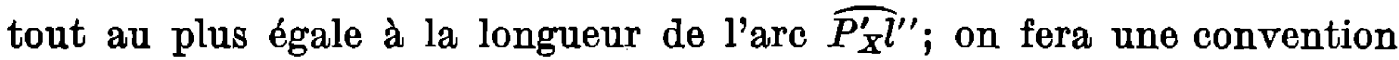
analogue si l'autre extrémité $l^{\prime \prime}$ se trouve sur la circonférence du cercle $K_{j_{1}}$.

Décomposons maintenant la seconde des intégrales (83) en une somme

$$
\Delta_{2}^{*}(X)=\Delta_{2}^{*\left(l^{\prime \prime} l^{\prime \prime}\right)}(X)+\Delta_{2}^{*\left(L-l^{\prime} l^{\prime \prime}\right)}(X)
$$

d'intégrales étendues à l'arc $\overbrace{}^{\prime} l^{\prime \prime}$ et à la partie restante $L-\overparen{l}^{\prime} l^{\prime \prime}$. En vertu de la troisième des inégalités (62), l'intégrale étendue à l'arc $\widehat{l}^{\prime} l^{\prime \prime}$ vérifie l'inégalité

$$
\begin{array}{r}
\left|\Delta_{2}^{*\left(l^{\prime} l^{\prime \prime}\right.}(X)\right|<\operatorname{const} \varkappa_{\Phi}\left[\int_{l^{\prime} P_{X}} \int_{X} \frac{\left|P_{X}^{\prime} Q\right|^{h_{\Phi}-1} d Q}{\left|C_{j} Q\right|^{\beta+h_{\varphi}}\left|P_{X} C_{j_{1}}\right|^{\beta+h_{\varphi}}}+\right. \\
\left.\quad+\int_{P_{X}^{\prime} l^{\prime \prime}} \frac{\left|P_{X}^{\prime} Q\right|^{h_{\Phi}-1} d Q}{\left|O_{j} P_{X}\right|^{\beta+h_{\Psi}}\left|Q C_{j_{1}}\right|^{\beta+h_{\varphi}}}\right] .
\end{array}
$$

Au nombre $\varepsilon$ on peat donc faire correspondre une longueur $\eta(\varepsilon)$ de l'are $\widehat{l^{\prime} l^{\prime \prime}}$ suffisamment petite pour qu'on ait

$$
\left|X P_{X}\right|^{\nu} \prod_{j=1}^{n}\left|X C_{j}\right|^{\beta+h_{\varphi}} \mid \Delta_{2}^{*\left(l^{\prime} t^{\prime \prime}\right)}(X)<\frac{\varepsilon}{4}
$$

quel que soit $X$ dans l'ensemble $\omega(\varepsilon)=S-\Sigma K_{j}(\varepsilon) \times S$. La seconde des intégrales (85) reste régulière, si $X$ tend vers $P_{X}^{\prime}$, donc au nombre $\varepsilon$ on peut faire correspondre un indice $N^{\prime}(\varepsilon)$ tel qu'on ait

$$
\left|X P_{X}\right|^{\gamma} \prod_{f=1}^{n}\left|X C_{f}\right|^{\beta+h_{\varphi}}\left|\Delta_{2}^{*\left(L-l \eta^{\prime \prime}\right)}(X)\right|<\frac{\varepsilon}{4}
$$


si $m>N^{\prime}(\varepsilon), X \in \omega(\varepsilon)$. En rapprochant les inégalités (81), (84), (87), (88), nous concluons que

$$
\lim _{m \rightarrow \infty} \sup \left[\left|X P_{X}\right|^{\nu} \prod_{j=1}^{n}\left|X C_{j}\right|^{\beta+h_{\varphi}}\left|\Delta_{2}(X)\right|\right]=0
$$

donc, en tenant compte du résultat (79), nous aurons

$$
\lim _{n \rightarrow \infty} \sup _{X \in S}\left[\left|X P_{X}\right|^{\gamma} \prod_{j=1}^{n}\left|X C_{j}\right|^{\beta+h_{\varphi}}\left|v_{\nabla}^{(m)}(X)-v_{p}(X)\right|\right]=0 .
$$

Il reste à étudier la différence $\psi^{(m)}(P)-\psi(P)$, où $\psi(P)$ est une solution de l'équation intégrale $(63)$ et $\psi^{(m)}(P)$ de l'équation analogue. On aura done

$$
\begin{gathered}
\psi^{(m)}(P)-\psi(P)=\left[\Xi\left(P, v_{0}^{(m)}, u_{0}^{(m)}, u_{1}^{(m)}, u_{2}^{(m)}\right)-\Xi\left(P, v_{0}, u_{0}, u_{1}, u_{2}\right)\right]+ \\
+\int_{L} \mathfrak{N}(P, Q)\left[\Xi\left(Q, v_{0}^{(m)}, u_{0}^{(m)}, u_{1}^{(m)}, u_{2}^{(m)}\right)-\Xi\left(Q, v_{0}, u_{0}, u_{1}, u_{2}\right)\right]
\end{gathered}
$$

où $\Xi$ est le résultat d'une opération fonctionnelle sur les fonctions $v_{0}, u_{0}, u_{1}, u_{2}$, définie par les termes intégrales de la seconde des relations (63). Il suffit d'étudier la différence des derniers termes, contenant des intégrales à singularité forte.

D'après l'hypothèse (9), nous aurons

$$
\left|G\left(P, v_{0}^{(m)}, \bar{u}_{s}^{(m)}\right)-G\left(P, v_{0}, \bar{u}_{s}\right)\right| \leqslant M_{G}\left[\left|v_{0}^{(m)}-v_{0}\right|+\left|\bar{u}_{s}^{(m)}-\bar{u}_{s}\right|\right]
$$

où les fonctions $\bar{u}_{s}(P)$ sont déterminées par les formules $\left(56^{\prime}\right)$ et les fonctions $\bar{u}_{s}^{(m)}(P)$ par des formules analogues. Moyennant des décompositions et des raisonnements analogues à ceux qui ont été faits pour les intégrales (77) et (83), on arrive à la même conclusion

$$
\lim _{m \rightarrow \infty} \sup _{P \in L}\left[\prod_{=-1}^{n}|P C|^{\beta+h_{\varphi}} \mid G\left(P, v_{0}^{(m)}, \bar{u}_{s}^{(m)}\right)-G\left(P, v_{0}, u_{s}\right)\right]=0 .
$$

Les autres composantes de l'opération $\Xi$ sont plus faciles à étudier et on aura de même

$$
\begin{aligned}
\lim _{m \rightarrow \infty} \sup _{P \in L}\left[\prod_{i=1}^{n}\left|P C_{v}\right|^{\beta+h_{\varphi}} \mid \Xi\left(P, v_{0}^{(m)}, u_{0}^{(m)}, u_{1}^{(m)}, u_{2}^{(m)}\right)-\right. & \\
& \left.-\Xi\left(P, v_{0}, u_{0}, u_{1}, u_{\dot{*}}\right) \mid\right]=0 .
\end{aligned}
$$

$\Pi$ en résulte, en vertu de la formule (91), la propriété suivante

$$
\lim _{m \rightarrow \infty} \sup _{P \in L}\left[\prod_{\nu=1}^{n}\left|P C_{\nabla}\right|^{\beta+h_{\varphi}}\left|\psi^{(m)}(P)-\psi(P)\right|\right]=0 .
$$


En rapprochant les résultats (90) et (95), on obtient

$$
\lim _{m \rightarrow \infty}\left\|\nabla^{(m)}-\nabla\right\|=0
$$

et par conséquent le lemme 1 est démontré.

LEMnE 2. L'ensemble $E^{\prime}$, transformé de l'ensemble $E$ par les rèlations (63), est compact.

Démonstration. Conformément à la définition de la compacité, il est nécessaire et suffisant de démontrer que de la suite arbitraire $\left\{\nabla^{m}\right\}$ de points $\nabla^{(m)}=\left[v_{0}^{(m)}, v_{1}^{(m)}, v_{2}^{(m)}, \psi^{(m)}\right]$ de l'ensemble $E^{\prime}$ on peut extraire une suite partielle $\left\{V^{\left(k_{m}\right)}\right\}$ convergente au sens de la norme (60).

Remarquons pour cela que, d'après les limitations (62), nous pouvons faire correspondre au nombre positif arbitraire $\varepsilon$ un rayon $r(\varepsilon)$ des cercles $K_{j}$, centrés en $C_{j}$, suffisamment petit pour qu'on ait (quel que soit $m$ )

$$
\prod_{j=1}^{n}\left|P C_{j}\right|^{\beta+h_{F}}\left|\psi^{(m)}(P)\right|<\frac{\varepsilon}{4}
$$

si le point $P$ est situé dans les cercles $K_{j}$. Les cercles $K_{j}(\varepsilon)$ étant fixés, les fonctions $\psi^{(m)}(P)$ sont bornées dans leur ensemble et également continues dans l'ensemble $L^{\prime}$ de tous les points de $L$ situés à l'extérieur des cercles $K_{j}$. Cette propriété est évidente pour l'ensemble de toutes les fonctions $\psi^{(m)}(P)$, puisqu'elles vérifient la même condition de Hölder (74).

En vertu de l'inégalité (66), nous pouvons maintenant faire correspondre à tout nombre positif $\varepsilon$ un ensemble fini de $q+q^{\prime}$ lignes fermées

$$
L_{1}^{s}, L_{2}^{s}, \ldots, L_{a+\alpha^{\prime}}^{s}
$$

situées à l'intérieur de l'ensemble $S$ d'un côté des lignes fermées $L_{0}, \ldots, L_{q}$ et entourant chacun des arcs $l_{1}, l_{2}, \ldots, l_{q^{\prime}}$, assez voisines de ces lignes pour que les fonctions $v^{(m)}(X)$ vérifient l'inégalité

$$
\left|X P_{X}\right|^{\gamma} \prod_{j=1}^{n}\left|X C_{j}\right|^{\beta+h_{\varphi}}\left|v_{\boldsymbol{r}}^{(m)}(X)\right|<\frac{\varepsilon}{4}
$$

en tout point $X$ de la partie $S_{\varepsilon}$ de l'ensemble $S$ limitée par les lignes (97). L'ensemble $\boldsymbol{S}-\boldsymbol{S}_{8}$ ne contient pas dans son intérieur les lignes $l_{1}, l_{2}, \ldots, l_{q^{\prime}}$ et dans cet ensemble les fonctions $\left|X P_{X}\right|$ et $\left|X C_{j}\right|$ ont des bornes inférieures positives. Donc les fonctions $v_{\nu}^{(m)}(X)$ sont continues dans la fermeture $\overline{S-S_{e}}$ de l'ensemble $S-S_{s}$ et, en vertu du théorème 5 , elles sont bornées dans leur ensemble et vérifient dans cet ensemble une même condition de Hölder (quel que soit $m$ )

$$
\left|v_{*}^{(m)}(X)-v_{z}^{(m)}\left(X_{1}\right)\right| \leqslant H_{\varepsilon}\left|X X_{1}\right|^{\theta} \text {. }
$$

En tenant compte des propriétés citées des fonctions $\psi^{(m)}(P)$ dans l'enensemble $L^{\prime}$ et des fonctions $v_{\eta}^{(m)}(X)$ dans l'ensemble $S-S_{\eta}$, nous con- 
cluons, d'après le théorème connu d'Arzelà, qu'il existe une suite $\left\{k_{m}\right\}$ d'indices telle que les séries partielles

$$
\left\{\psi^{\left(k_{m}\right)}(P)\right\}, \quad\left\{v_{v}^{\left(k_{m}\right)}(X)\right\}
$$

soient uniformément convergentes, au sens habituel, pour $P \in L^{\prime}$, resp. $X \in S-S_{8}$. Nous pouvons donc faire correspondre à tout nombre positif $\varepsilon$ un indice $N_{e}$, tel qu'on ait

$$
\begin{aligned}
& \sup _{P \in L}\left[\prod_{j=1}^{n}\left|P C_{j}\right|^{\beta+h_{\varphi}}\left|\psi^{\left(k_{m}\right)}(P)-\psi^{\left(k_{*}\right)}(P)\right|\right]<\frac{\varepsilon}{2} \\
& \max _{\bullet} \sup _{X \in S-S_{e}}\left[\left|X P_{X}\right|^{\gamma} \prod_{j=1}^{n}\left|X C_{j}\right|^{\beta+h_{\varphi}}\left|v_{v}^{\left(k_{m}\right)}(X)-v_{\nu}^{k_{s}}(X)\right|\right]<\frac{\varepsilon}{2}
\end{aligned}
$$

si $m, s>N_{\varepsilon}$. En rapprochant les inégalités (96), (98) (100), nous concluons que

$$
\left\|V^{\left(k_{m}\right)}-\nabla^{\left(k_{\varepsilon}\right)}\right\|<\varepsilon
$$

si $m, s>N_{s}$. Or, l'espace $A$ étant complet, cette condition de Cauchy est à la fois nécessaire et suffisante pour la convergence de la suite partielle $\left\{V^{\left(k_{m}\right)}\right\}$ au sens de la norme (60). Le lemme 2 est donc démontré.

En s'appuyant sur les deux lemmes démontrés, nous concluons, d'après le théorème cité de Schauder, qu'il existe dans l'ensemble $E$ au moins un point $U^{*}=\left[u_{0}^{*}, u_{1}^{*}, u_{2}^{*}, \varphi^{*}\right]$ invariant relativement à la transformation (63), donc donnant une solution du système (56) d'équations intégrales. En vertu des propriétés des potentiels généralisés (17) et (24), nous avons les relations

$$
u_{1}^{*}(X)=\frac{\partial u_{0}^{*}}{\partial x_{1}}, \quad u_{2}^{*}(X)=\frac{\partial u_{0}^{*}}{\partial x_{2}}
$$

en tout point $X\left(x_{1}, x_{2}\right)$ de l'ensemble $S$, donc les fonctions $u_{0}^{*}(X), \varphi^{*}(P)$ vérifient le système d'équations intégro-différentielles (53), (55).

Observons maintenant, en vertu des hypothèses (2), (5) et le théorème 5 , que la fonction

$$
\Phi(\boldsymbol{Y})=F_{1}\left[Y, u_{0}^{*}(\bar{Y}), u_{1}^{*}(\bar{Y}), u_{2}^{*}(\boldsymbol{Y})\right]
$$

vérifie une condition de Hölder dans tout domaine fermé $S^{*}$ situé à l'intérieur de l'ensemble $S$, donc, d'après le théorème 6 , la fonction trouvée $u_{0}^{*}(Y)$ vérifie l'équation différentielle donnée (1) en tout point $X$ de l'ensemble $S$. D'après l'équation (55) et le théorème 1 , cette fonction et ses dérivées ont des valeurs limites qui vérifient la condition demandée (10) en tout point $P$ sur $L$ différent des points de discontinuité $C_{*}$. En outre, en vertu $d u$ théorème 4 , la fonction $u_{0}^{*}$ vérifie la condition (13). Nous pouvons donc énoncer le théorème suivant. 
THEork̀me 7. Si les conditions I-VII, précisées précédemment, sont verifiées, si les constantes $M_{F}, M_{F}^{\prime}, M_{G}, M_{G}^{\prime}, M_{\sigma}, k_{G}, k_{\sigma} d u$ problème sont suffisamment petites pour que les inégalités (75) soient vérifiées, il existe au moins une fonction $u(X)$ qui: 1) vérifie l'équation elliptique (1) en tout point $X$ de l'ensemble $S, 2)$ vérifie la condition limite aux derivées tangentielles (10) en tout point $P \in L$, différent des points $\left.C_{r}, 3\right)$ vérifie la condition (13).

\section{Travaux cités}

[1] H. Poincaré, Mécanique céleste, III, Paris 1910.

[2] B. H védélidzé, Sur le problème aux limites de Poincaré (en russe), C. R. AcadSSSR. 30 (1941), p. 195-198.

[3] J. N. Vécoua, Problèmes aux limites dans la théorie des équations elliptiques (en russe), C. R. Ac. SSSR. 1940.

[4] N. J. Muskhelishvili, Singular integral equations, Groningen, Holland, 1953, p. 210.234.

[5] W. Pogorzelski, Problème aux limiles de Poincaré généralisé, Ann. Pol. Mathem. 2 (1955), p. 257.270.

[6] - Problème aux limites aux dérivées tangentielles pour l'équation elliptique, Bull. Acad. Pol. Sc, Sér. des sci. math., astr. et phys. 7 (1959), p. 205-212.

[7] - Problème aux limites aux dérivées tangentielles pour l'équation parabolique, Ann. Sc. Ec. Norm. Sup., Paris 1958, p. 19-35.

[8] - Sur la résolution du problème aux dérivées tangentielles pour l'équation parabolique par la méthode des approximations successives, Ann. Pol. Mathem. 7 (1959), p. 93-112.

[9] - Problemes aux limites discontinus dans la théorie des fonctions analytiques, Bull. Ac. Pol. Sc., Sér. des sci. math., astr. et phys. 7 (1959), p. 311-317.

[10] M. Gevrey, Détermination et emploi des fonctions de Green, Journal de Mathématiques, Paris 1930.

[11] G. Girand, Sur le problème de Dirichlet généralisé, Ann. Ec. Norm. Sup., Paris, 46 (1929), p. 131-245.

[12] W. Pogorzelski, Etude de la solution fondamentale de l'équation elliptique et des problèmes aux limites, Ann. Pol. Mathem. 3 (1957), p. 247-284.

[13] - Propriétés des dérivées tangentielles d'une intégrale de l'équation elliptique, Ann. Pol. Mathem. 7 (1960), p. 321-339.

[14] - Sur l'équation intégrale singulière et sur les propriétés d'une intégrale singulière, Journal of Mathem. and Mechanics, Indiana Univ. 1958, p. 515-532.

[15] - Problemes aux limites discontinus dans la théorie des fonctions analytiques, Bull. Acad. Pol. Sc., Sér. des sci. math., astr. et phys., 7 (1959), p. $711-714$.

[16] - Sur une propriété principale d'une classe $\mathfrak{\square}$ de fonctions discontinues pour le système d'arcs, Bull. Acad. Pol. Sc., Sér. des sci. math., astr. et phys. 8 (1960), p. 359-364.

[17] J. Schauder, Der Fixpunktsatz in Funktionalräumen, Studia Mathematica 2 (1930), p. 171-180.

INSTYTUT MATEMATYCZNY POLSKIEJ AKADEMII NAUK INSTITUT MATHEMATIQUE DE L'ACADEMIE POLONAISE DES SCIENCES 